\title{
THE NATURE OF THE EMISSION TRANSITION OF THE OCTAHEDRAL URANATE GROUP
}

\author{
J.Th.W. DE HAIR and G. BLASSE \\ Salid State Chemistry Department, Physical Laboratory, State University. Utrecht. The Netherlands
}

Received 27 June 1975

Decay times of the green luminescence of $\mathrm{U}^{6+}$ in oxides with perovskite structure are reported. The influence of the site symmetry is found to be considerable. The tempetature dependence of the decay time gives evidence for a coupling of the electronic transition with a phonon of about $200 \mathrm{~cm}^{-1}$ in the excited state. The results show the emission to originate from a parity-forbicien transition, vibronically allowed by coupling with vibrations of ungerade symmetry.

\section{Introduction}

Recently we have reported the luminescence of hexavalent uranium in oxides with ordered perovskite structure [1]. These compounds show an efficient green luminescence. At present a more detailed study of the luminescence mechanism has been carried out. In this note we report on the nature of the transition responsible for the emission.

Up to now only the electronic transitions in the uranyl ion ( $\mathrm{UO}_{2}^{2+}$ ) have been discussed thoroughly [2,3]. In general its emission is assumed to be a spinforbidden triplet-singlet transition [2]. Jórgensen, however, has argued that it has no meaning to speak about singlet or triplet states [4]. For a fluorescing level of $\mathrm{CaF}_{2}$ : $\mathrm{U}^{6+}$ Manson et al. have measured the Zeeman splitting [5]. Their analysis shows the zerophonon emission line to be a pure magnetic dipole transition. The emitting centre has been shown to have threefold symmetry. From the absence of electricdipole intensity it is assumed to have a centre of symmetry. They could not decide definitely between a $\mathrm{UO}_{2}^{2+}$ and a $\mathrm{UO}_{6}^{6-}$ group as the emitting centre.

We are considering the luminescence of the $\mathrm{NO}_{6}^{5-}$ group in oxides with ordered perovskite structure. The emitting centre in this type of compounds occupies a site with inversion symmetry. As mentioned earlier this emission shows vibrational fine structure at low temperatures [1]. The vibronic lines can be assigned to a coupling of the electronic transition with ungerade vibrational modes [0]. Together with the long decay time reported before, this points to a parityforbidden electric-dipole transition.

The parity selection rule can be relaxed by a permanent non-centrosymmetric distortion or by coupling with ungerade vibrational modes. In this note the influence of the former is considered by studying the luminescence decay in a series of compounds with increasing deviation from cubic site symmetry, whereas the influence of the latter is found from the temperature dependence of the decay time.

\section{Experimental}

We have measured the decay time of SrLaNaW $0.997 \mathrm{U}_{0.003} \mathrm{O}_{6}$ and of compositions with the general formula $\mathrm{A}_{2} B W_{0.997} \mathrm{U}_{0.003} \mathrm{O}_{6}(\mathrm{~A}=\mathrm{Ca}, \mathrm{Sr}$, $\mathrm{Ba} ; \mathrm{B}=\mathrm{Mg}, \mathrm{Ca}, \mathrm{Sr}, \mathrm{Zn}$ ). Powder samples were prepared and checked as described before [1]. SrLaNaW $997 \mathrm{U}_{0.003} \mathrm{O}_{6}$ was fired at $900^{\circ} \mathrm{C}$ for about 7 hours. This is a new compound which is related to a series of ordered perouskites with cubic crystal struc: ture reported by Brixner [7]. In contrast with these $\mathrm{SrLaNaWO}_{6}$ has an orthorombic structure. The lattice parameters are $a=5.80 \AA, b=5.67 \AA$ and $c=8.08 \AA$.

The samples were excited with a pulsed Xe-lamp (pulse width $\pm 1, t \mathrm{~s}$ ), along with a $366 \mathrm{~nm}$ Balzers narzuw oand interferesce filter. By using this combination we excite directly into the uranate group. The relevant 
Table 1

Decay time of the luminescence of $U^{6+}$ in oxides with perovskitc structure at LNT. Mainly $366 \mathrm{~nm}$ excitation. All compounds contain 0.3 mol percent $\mathrm{U}^{\mathrm{i}+}$

\begin{tabular}{llllll}
\hline $\mathrm{Ba}_{2} \mathrm{MgWO}_{6}$ & $255 \mu \mathrm{s}$ & $\mathrm{Ba}_{2} \mathrm{MgWO}_{6}$ & $255 \mu \mathrm{s}$ & $\mathrm{SrLaNaWO}_{6}$ & $\leqslant 155 \mu \mathrm{s}$ \\
$\mathrm{Ba}_{2} \mathrm{ZnWO}_{6}$ & $260 \mu \mathrm{s}$ & $\mathrm{Sr}_{2} \mathrm{MgWO}_{6}$ & $245 \mu \mathrm{s}$ & $\mathrm{Ba}_{2} \mathrm{Li}_{1.2} \mathrm{~W}_{0.8} \mathrm{O}_{6}$ & 10 and $80 \mu \mathrm{s}\{12\}$ \\
$\mathrm{Ba}_{2} \mathrm{CaWO}_{6}$ & $240 \mu \mathrm{s}$ & $\mathrm{Ca}_{2} \mathrm{MgWO}_{6}$ & $220 \mu \mathrm{s}$ & & \\
$\mathrm{Ba}_{2} \mathrm{SrWO}_{6}$ & $200 \mu \mathrm{s}$ & & & & \\
\hline
\end{tabular}

absorption is an allowed transition. In the emission beam a Balzers broad band interference $\mathrm{K} 3$ filter was placed, which has a maximum transmission for the emission. The emission intensity was measured with a photomultiplier and the time dependence was displayed on the screen of a sampling oscilloscope. For averaging and storing of the signal use was made of a waveform eductor.

For $\mathrm{Ba}_{2} \mathrm{MgW}_{0.997} \mathrm{U}_{0.003} \mathrm{O}_{6}$ we have measured the temperature dependence of the decay time. The sample was mounted in a liquid helium flow cryostat (Oxford CF 100). The temperature could be adjusted between liquid HeT and RT by varying the flow rate.

The decay time of the other samples was measured at LNT. We used a home-made cryostat, which was cooled by thermal contact with a liquid nitrogen bath.

\section{Results}

In table 1 decay times are given for numerous compositions at LNT. The decay curves can be described by single exponentials. Only for $\mathrm{SrLaNaW}_{0.997} \mathrm{U}_{0.003} \mathrm{O}_{6}$ a sum of several exponentials gives a better agreement with the experimental curve. The longest decay time was $155 \mu$, the others were shorter.

The temperature dependence of the decay time of $\mathrm{Ba}_{2} \mathrm{MgW}_{0.997} \mathrm{U}_{0.003} \mathrm{O}_{6}$ is given in fig. 1 together with the temperature dependence of the emission intensity. Note that the decay time decreases in a region where the emission intensity is constant.

\section{Discussion}

The emission of the compounds studied shows vibrational fine structure at lower temperatures. We can distinguish a well-defined one-phonon part. It follows from our assignment that the vibrational modes involved are the ungerade internal modes of the $\mathrm{UO}_{6}^{6-}$

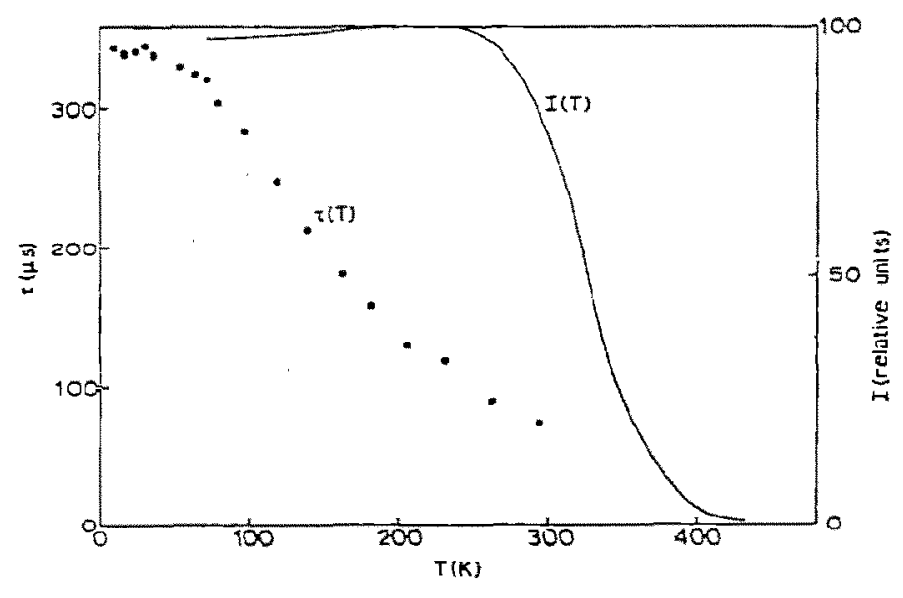

Fig. 1. Temperature dependence of the decay time $t$ and the intensity $I$ of the green emission of $\mathrm{Ba}_{2} \mathrm{MgW}_{0.997} \mathrm{U}_{0.003} \mathrm{O}_{6}$. Mainly $366 \mathrm{~nm}$ excitation.

group [6]. Vibrational spectroscopic studies of compounds with orciered perovskite structure have shown that we may consider the $\mathrm{UO}_{6}^{6-}$ group as an isolated molecular group with its own internal vibrational modes, corresponding with those for an octahedron $[8,9]$. For such a group there are three modes with "ungerade" symmetry, viz. $\nu_{3}\left(\mathrm{~T}_{1 \mathrm{u}}\right), \nu_{4}\left(\mathrm{~T}_{1 \mathrm{u}}\right)$, and $\nu_{6}\left(T_{2 u}\right)$. Coupling of the pure electronic transition with one of the three ungerade modes is an indication for a parity-forbidden electric-dipole transition.

A static removal of the centre of symmetry can also relax the parity selection rule. For the given compounds vibrational spectrosccpic results are available [10]. From these we learned that an increasing broadening of the two IR active bands appeared from top to bottom in table 1. A broadening of these bands is due to a deviation from cubic symmetry which lifts the threefold degeneracy of these modes. A deviation from inversion symmetry may be the result. We see from table 1 that in the series $\mathrm{A}_{2} \mathrm{BWO}_{6}-\mathrm{U}$ the decay time decreases with increasing deviation from cubic symmetry. 
Note that the measurements were made for a concentration where concentration quenching is negligible and at a temperature where temperature dependent radiationless decay can be neglected [6].

For $\mathrm{S}_{\mathrm{L}} \mathrm{LNaW} 0.997 \mathrm{U}_{0.003} \mathrm{O}_{6}$ we have found more than one decay time. This is the result of the presence of $\mathrm{UO}_{6}^{6-}$ groups with different surroundings. In the IR spectrum of this compound a weak additional absorption band is observed at the same frequency where the $\nu_{1}\left(A_{I g}\right)$ is observed in the Raman spectrum. This points to the mixed occupation of the larger cation sites by $\mathrm{Sr}^{2+}$ and $\mathrm{La}^{3+}$ ions which causes a deviation from inversion symmetry at the site of the hexavalent ion [11]. A great variety of luminescent centres with different surroundings is the result. This explains the relatively short decay times and the occurrence of more than one decay time.

It is interesting to note that in $\mathrm{Ba}_{2} \mathrm{Li}_{1.2} \mathrm{~W}_{0.8} \mathrm{O}_{6}-\mathrm{U}$, a compound with perovskite-like structure, two decay times have been found, viz. 10 and $80 \mu$ s [12]. Due to the sequence of the $\mathrm{BaO}_{3}$ layers in this crystal structure here are two different sites for the hexavalent metal ions, both with site symmetry $C_{3 v}$ These short decay times extend the relation of table 1 .

Further evidence for the presence of a parity-forbidden transition can be gained from the temperature dependence of the decay time. First of all one should note that the influence of the temperature on the decay time differs markedly from that on the luminescence intensity (fig. 1). While the latter is still constant, the former decreases. The excitation takes place by an allowed electronic transition. The slight temperature dependence of this process can be neglected. With increasing temperature the occupation of vibrational levels in the excited electronic state increases. The possibility for coupling with an ungerade vibration in the excited state becomes larger. The parityselection rule will be more relaxed dynamically and a decrease of the decay time is the result. The temperature dependence of the transition probability for a vibronically allowed transicion is contained in a factor $\left[\exp \left(h v_{\mathrm{a}} / k T\right)-1\right]^{-1}$, as described by Di Bartolo [13]. Here $\nu_{\mathrm{a}}$ is the fiequency of the phonon which is involved in the coupling.

Analysis of the results with this relationship gives a very good agreement for temperatures below $120 \mathrm{~K}$.
We have succeeded in a fit using a $v_{a}$ of about $200 \mathrm{~cm}^{-1}$. This ungerade vibration is probably the $\nu_{6}\left(T_{2 u}\right)$ internal mode. This vibration is IR inactive. The frequency of this mode found from the vibrational pattern in the emission of $\mathrm{Sr}_{2} \mathrm{MgW}_{0.997} \mathrm{U}_{0.003} \mathrm{O}_{6}\left(240 \mathrm{~cm}^{-1}\right)$ is a little higher than the $\nu_{a}$ frequency [6]. This difference can be explained by the fact that the latter corresponds to a vibrational mode in the excited state.

In conclusion we may state that we have given strong evidence for a parity-forbidden transition in the emission of the $\mathrm{UO}_{6}^{6-}$ group. It is possible that for the $\mathrm{UO}_{2}^{2+}$ group the electronic transition responsible for the luminescence has to be considered in the same way.

\section{Acknowledgement}

The investigations were performed as a part of the research program of the "Stichting voor Fundamenteel Onderzoek der Materie" (F.O.M.) with financial support from the "Nederlandse Organisatie voor Zuiver Wetenschappelijk Onderzoek" (Z.W.O.)

\section{References}

[1] J.Th.W. de Hair and G. Blasse, J. Luminescence 8 (1973) 97.

[2] S.P. McGlynn and J.K. Smith, J. Mol. Spectry. 6 (196 I) 164.

[3] H.D. Burrows and T.J. Kemp, Chem. Soc. Rev. (1974) 139.

[4] C.K. J $\$$ rgensen, Modern aspects of Iigand ficld theory (North-Holland, Amsterdan, 1971) p. 503.

[5] N.B. Manson, G.A. Shah and W.A. Runciman, Solid State Commun. 16 (1975) 645.

[6] J.Th.W. de Hair, to be publiched.

[7j L.H. Bixner, Mat. Res. Bull. 9 (1974) 1041.

[8] G. Blasse and A.F. Corsmit, J. Solid State Chem. 6 (1973) 513.

[9] A.F. Corsmit, H.E. Hoefdraad and G. Blasse, J. Inorg. Nucl. Chem. 34 (1972) 3401 .

[10] J.Th.W. de Hair, unpublished results.

[11] D. Krol anci G. Blasse, J. Inorg. Nucl. Chem. 37 (1975) 1328.

[12] G. Blasse, J. Solid State Chem. 14 (1975), to be published.

[13] B. Di Bartolo, Optical interactions in solids (Wiley, New York, 1967). 